\title{
Workshop on Agent-Based Modeling
}

After the success of the first workshop of Agent-based approach in Economic and Social Complex Systems (AESCS) in Shimane, 2001, we have held a similar workshop in Niigata, 2003. The title is International Workshop on Agent-Based Modeling. The aims and scope are summarized as follows: Agent-Based Modeling has become one of major techniques to design and analyze complex adaptive systems including societies, economics, organizations, business management, Web applications, and the other engineering fields. The objective of the Workshop is to continue the efforts to foster the formation of an active multi-disciplinary community on multiagent, computational economics, organizational science, social dynamics, and complex adaptive systems, in conjunction with the 17th annual conference of JSAI, the largest AI related annual conference in the Pacific Asia region.

At the workshop, we have had twelve oral presentations. They were pearly reviewed after the workshop, and then we have decided to accept the following five papers for the inclusion of these post-proceedings: PjS. Tomita, A. Namatame: Bilateral Trading with and without Strategic Thinking QjK. Miyanishi, K. Suzuki: Cooperative Behavior with Common Information Controller in Minority Game RjK. Izumi: Analysis of Efficiency and Accuracy of Learning in Minority Game SjK. Yuta et al.: Sectioned Random-Network Agent Model Demonstrates Behavior of Sectionalism TjM. Kunigami, T. Terano: Interaction and Control in Learning Agent System

The selected papers cover the wide area of agent-based modeling and have shown the effectiveness of the approach to both theoretical and practical issues of the domains.

Following to the workshop, we are continue to have the AESCS workshop series in 2002, 2004 and 2005, for Pacific Asian regions. Furthermore, in August 2006, integrating European, American, and Pacific-Asian academic associations, we held the First World Congress on Social Simulation in Kyoto. The postproceedings will be also published from Springer Verlag, very soon in 2007. We believe that agent-based modeling for social complex systems is quite promising for the future Artificial Intelligence researches. 